\title{
ATTRACTION REGION OF PLANAR LINEAR SYSTEMS WITH ONE UNSTABLE POLE AND SATURATED FEEDBACK
}

\author{
J.-Y. FAVEZ, PH. MULLHAUPT, B. SRINIVASAN, and D. BONVIN
}

\begin{abstract}
The bifurcation of the attraction region for planar systems with one stable and one unstable pole under a saturated linear state feedback is considered. The attraction region can have either an unbounded hyperbolic shape or be bounded by a limit cycle. An analytical condition, under which either of these boundary shapes occurs, is given with a formal proof. This condition is based on the relationship between the stable and unstable manifolds associated with secondary saddle equilibrium points, whose presence is caused by the saturation on the input.
\end{abstract}

\section{InTRODUCTION}

The study, whose results are presented here, originates from the problem of stabilizing a tokamak plasma reactor $[6,7]$. For small excursions around a nominal set point, the model to be controlled is considered as having only one unstable pole and a large number of stable ones. The main difficulty is the presence of the input saturation due to the voltage and current limitation. Additionally, the specificity of the control hardware at the user disposal (at the plant location) is of practical importance. The hardware can implement only linear feedbacks, since no more than matrix multiplications and some extra simple algebra are allowed.

The importance of the effect of saturation on the limitation of the stability region is observed experimentally using linear feedbacks, which encourages a sound theoretical treatment of the underlying issues. One key question is the impact of the gains on the shape and size of the stability region. However, such a task (in its full generality) is daunting. Hence, one is naturally conducted to study the simplest system retaining most of the main characteristics, namely a planar system having one stable and one

2000 Mathematics Subject Classification. 34C23, 93C10, 34D99.

Key words and phrases. Planar linear system, input saturation, attraction region, bifurcation. 
TABLE 1. Characteristics of the attraction regions

\begin{tabular}{||l|l||}
\hline Pole configuration & Attraction region \\
\hline Both stable & $\mathbb{R}^{2}$ \\
One stable, one unstable & bifurcation \\
Both unstable & closed by a limit cycle \\
\hline
\end{tabular}

unstable pole under a saturated feedback, the results of which are presented hereafter.

Nevertheless, and for general planar systems (i.e., nonlinear) under an input saturation, maximum stabilizing feedbacks that guarantee the state convergence in minimum time can also be constructed $[2-5,14,15]$. However, such feedbacks are not perfectly appropriate in the present context, since, apart from the implementation constraints mentioned above, they are very sensitive to switching time instants. Indeed, inaccurate state measurements and model uncertainty (both of which cannot be avoided) can give a poor performance. This robustness issue is not so crucial when simple linear feedbacks are considered.

Two important concepts pertaining to systems under a saturated input have to be distinguished. First is the null controllable region, i.e., the region in the state space where there exists an input that can steer the system to the origin $[1,8,9,12]$. Second is the attraction region with a given controller, i.e., the region in the state space, from which the closed-loop system asymptotically reaches the origin $[1,8]$. In this paper, only the issues belonging to the latter, i.e., the attraction region, will be studied. Also, the design of controllers for which the attraction region is arbitrarily close to the null controllable region [11] will not be studied here. The interested reader can consult $[2,4]$ (and references therein) for such a complementary treatment. Single input linear planar systems (systems with two states) with saturated linear feedbacks will be considered. It will be assumed that the linear feedback makes the origin globally asymptotically stable in the absence of the saturation.

The shape of the attraction region depends on the location of the openloop poles. If both poles are stable or semi-stable, then the system is globally stabilizable $[1,16]$. If both poles are unstable, then the boundary of the attraction region is a closed trajectory [1]. A method for finding this closed trajectory (limit cycle) is provided in [8,9]. For systems with one stable and one unstable pole, it has been shown in [1] that the topological bifurcation of the attraction region takes place, i.e., the attraction region changes as a function of the system and controller parameters from the hyperbolic type to a limit cycle. The characteristics of the attraction region are summarized in Table 1. 
Since this paper deals with bifurcations, only the case with one stable and one unstable pole will be considered. Although this problem is studied in [1], the result therein does not characterize the bifurcation. An analytical condition, under which a bifurcation occurs, is derived in [7]. The main contribution of the present paper is the qualitative description of the attraction region and the explicit proof of the existence of different attraction regions and the condition under which they occur.

The paper is organized as follows. In Sec. 2, definitions and notation used in the paper are introduced. An important result concerning the equilibrium points of the closed-loop system is provided. Section 3 provides the bifurcation condition. In Sec. 4, the shape of the attraction region is given as a function of this condition. Finally, conclusions are drawn in Sec. 5.

\section{Preliminaries}

2.1. Linear planar system. Consider a single-input second-order linear system with one stable and one unstable pole. Upon the state transformation, the system can be written as a decoupled system, i.e.,

$$
\dot{\hat{x}}=\hat{A} \hat{x}+\hat{b} u=\left[\begin{array}{cc}
\lambda_{1} & 0 \\
0 & \lambda_{2}
\end{array}\right] \hat{x}+\left[\begin{array}{l}
\hat{b}_{1} \\
\hat{b}_{2}
\end{array}\right] u,
$$

where $\hat{x} \in \mathbb{R}^{2}$ is the state vector, $u$ is the input, $\hat{A}$ and $\hat{b}$ are appropriate matrices, and $\lambda_{1}$ and $\lambda_{2}$ are the eigenvalues of the system. Assume that $\lambda_{1}>0$ and $\lambda_{2}<0$. Furthermore, we scale the input by considering the state transformation

$$
x=T \hat{x}=\left[\begin{array}{cc}
\lambda_{1} / \hat{b}_{1} & 0 \\
0 & \lambda_{2} / \hat{b}_{2}
\end{array}\right],
$$

which leads to the orthogonalized and normalized system

$$
\dot{x}=A x+b u=\left[\begin{array}{cc}
\lambda_{1} & 0 \\
0 & \lambda_{2}
\end{array}\right] x+\left[\begin{array}{l}
\lambda_{1} \\
\lambda_{2}
\end{array}\right] u .
$$

Consider the symmetric saturation function with the unit saturation level:

$$
\operatorname{sat}(s)= \begin{cases}-1 & \text { if } \quad s<-1 \\ s & \text { if } \quad-1 \leq s \leq 1, \\ 1 & \text { if } \quad s>1\end{cases}
$$

With a saturated linear state feedback, the closed-loop system is

$$
\dot{x}=A x+b \operatorname{sat}(f x),
$$

where

$$
f=\left[\begin{array}{ll}
f_{1} & f_{2}
\end{array}\right] \in \mathbb{R}^{1 \times 2}
$$

is the feedback gain vector. The matrix $(A+b f)$ is assumed to be a Hurwitz matrix, i.e., the system is stable without saturation. Let $\tilde{\lambda}_{1}$ and $\tilde{\lambda}_{2}$ be the 
eigenvalues of $(A+b f)$. The two conditions that correspond to the case where $(A+b f)$ is Hurwitz are as follows:

$$
\begin{gathered}
\lambda_{1}\left(1+f_{1}\right)+\lambda_{2}\left(1+f_{2}\right)<0, \\
\lambda_{1} \lambda_{2}\left(1+f_{1}+f_{2}\right)>0 .
\end{gathered}
$$

Since $\lambda_{1} \lambda_{2}<0$, condition (2.7) becomes $\left(1+f_{1}+f_{2}\right)<0$. Also, it can be verified that $f_{1}<0$, while $f_{2}$ can take either sign.

\subsection{Equilibrium points and the attraction region.}

Theorem 2.1. The closed-loop system (2.5) has three equilibrium points:

$$
x_{e+}=A^{-1} b=\left[\begin{array}{ll}
1 & 1
\end{array}\right]^{T}, \quad x_{e-}=-A^{-1} b=\left[\begin{array}{ll}
-1 & -1
\end{array}\right]^{T}, \quad x_{e 0}=0 .
$$

Of these, $x_{e 0}$ is stable, while the other two are saddle points.

Proof. The theorem is a direct consequence of the eigenvalue analysis $[1$, 7].

Definition 2.2. Let $\Phi\left(t, x_{0}\right)$ denote the state of $(2.5)$ at the time $t$, starting from the initial condition $x_{0}$ at $t=0$. The attraction region of the stable equilibrium point is defined as follows:

$$
\mathcal{A}=\left\{x: \lim _{t \rightarrow \infty} \Phi(t, x)=0\right\} .
$$

The boundary of $\mathcal{A}$ is denoted by $\partial \mathcal{A}$.

2.3. Manifolds. Two important regions for the following construction should be distinguished. First, the null controllability region $\mathcal{C}$ corresponds to the set of states, for which there exists an open loop input that brings the state to zero (despite the saturation on this input). It is the vertical strip in the state space, whose boundaries are

$$
\partial \mathcal{C}_{+}=\left\{x \in \mathbb{R}^{2}: x_{1}=1\right\}, \quad \partial \mathcal{C}_{-}=\left\{x \in \mathbb{R}^{2}: x_{1}=-1\right\} .
$$

Second, the null reachability region is denoted by $\mathcal{R}$ and corresponds to all states that can be reached from the origin. It is a horizontal strip, whose boundaries are given by

$$
\partial \mathcal{R}_{+}=\left\{x \in \mathbb{R}^{2}: x_{2}=1\right\}, \quad \partial \mathcal{R}_{-}=\left\{x \in \mathbb{R}^{2}: x_{2}=-1\right\} .
$$

The interplay between the following straight lines and manifolds (whose origins, apart from $\partial \mathcal{L}_{0}$, are also connected with the saturation) is of the prime importance (refer to Fig. 1 for illustration):

(i) $\partial \mathcal{L}_{0}=\left\{x \in \mathbb{R}^{2}: f x=0\right\}$;

(ii) $\partial \mathcal{L}_{+}=\left\{x \in \mathbb{R}^{2}: f x=1\right\}, \partial \mathcal{L}_{-}=\left\{x \in \mathbb{R}^{2}: f x=-1\right\}$;

(iii) $\mathcal{S}\left(x_{e+}\right)=\left\{x \in \mathbb{R}^{2}: \lim _{t \rightarrow \infty} \Phi(t, x)=x_{e+}\right\}$;

(iv) $\mathcal{S}\left(x_{e-}\right)=\left\{x \in \mathbb{R}^{2}: \lim _{t \rightarrow \infty} \Phi(t, x)=x_{e-}\right\}$; 
(v) $\mathcal{U}\left(x_{e+}\right)=\left\{x \in \mathbb{R}^{2}: \lim _{t \rightarrow \infty} \Phi(-t, x)=x_{e+}\right\}$;

(vi) $\mathcal{U}\left(x_{e-}\right)=\left\{x \in \mathbb{R}^{2}: \lim _{t \rightarrow \infty} \Phi(-t, x)=x_{e-}\right\}$.

The straight lines $\partial \mathcal{L}_{+}$and $\partial \mathcal{L}_{-}$are the boundaries of the region $\mathcal{L}$, where the control is linear. $\partial \mathcal{L}_{0}$ is the straight line corresponding to the zero control. $\mathcal{S}\left(x_{e \pm}\right)$ and $\mathcal{U}\left(x_{e \pm}\right)$ denote the stable and unstable manifolds of the equilibrium saddle points, respectively. As concerns the unstable manifolds, evolution in reverse time is considered. All manifolds have two branches, both of which start from the equilibrium point and move out in different directions.

\section{Bifurcation of the ATtraction Region}

3.1. Existence of a bifurcation. For a system with one stable and one unstable open-loop pole and a saturated (linear or nonlinear) state feedback, a result on the existence of a bifurcation of the attraction region is given in [1]:

1. If $\mathcal{U}\left(x_{e+}\right) \cap \mathcal{A} \neq \emptyset$ and $\mathcal{U}\left(x_{e-}\right) \cap \mathcal{A} \neq \emptyset$, then $\partial \mathcal{A}=\mathcal{S}\left(x_{e+}\right) \cup \mathcal{S}\left(x_{e-}\right)$.

2. If $\mathcal{U}\left(x_{e+}\right) \cap \mathcal{A} \neq \emptyset$ and $x_{e-} \notin \partial \mathcal{A}$, then $\partial \mathcal{A}=\mathcal{S}\left(x_{e+}\right)$.

3. If $\mathcal{U}\left(x_{e-}\right) \cap \mathcal{A} \neq \emptyset$ and $x_{e+} \notin \partial \mathcal{A}$, then $\partial \mathcal{A}=\mathcal{S}\left(x_{e-}\right)$.

4. If $\mathcal{U}\left(x_{e+}\right) \cap \mathcal{A}=\emptyset$ and $\mathcal{U}\left(x_{e-}\right) \cap \mathcal{A}=\emptyset$, then $\partial \mathcal{A}$ is either a closed orbit or a graph of homoclinic/heteroclinic connections.

This result calls for some remarks. First, the result depends on the shape of $\mathcal{A}$ and $\partial \mathcal{A}$, both of which are unknown. Second, since system (2.5) is symmetric, items 2 and 3 cannot happen. Furthermore, homoclinic connections (manifolds starting from and ending at the same saddle point) do not exist for the system considered. However, it is possible to distinguish between the cases, where

(i) heteroclinic connections occur (manifolds starting from one saddle point and ending at another saddle point) and

(ii) a closed orbit appears.

3.2. Connection between the stable manifold and the shape of the attraction region. Starting from the saddle points, we can extend the branches of the manifolds $\mathcal{S}\left(x_{e \pm}\right)$ and $\mathcal{U}\left(x_{e \pm}\right)$ along $\partial \mathcal{C}_{ \pm}$and $\partial \mathcal{R}_{ \pm}$until they enter the linear region. The corresponding intersection points are

$$
\begin{aligned}
& c_{+}=\partial \mathcal{C}_{-} \cap \partial \mathcal{L}_{+}=\left[\begin{array}{ll}
-1 & \frac{\left(1+f_{1}\right)}{f_{2}}
\end{array}\right]^{T} \\
& r_{-}=\partial \mathcal{R}_{+} \cap \partial \mathcal{L}_{-}=\left[\begin{array}{ll}
-\frac{\left(1+f_{2}\right)}{f_{1}} & 1
\end{array}\right]^{T} .
\end{aligned}
$$



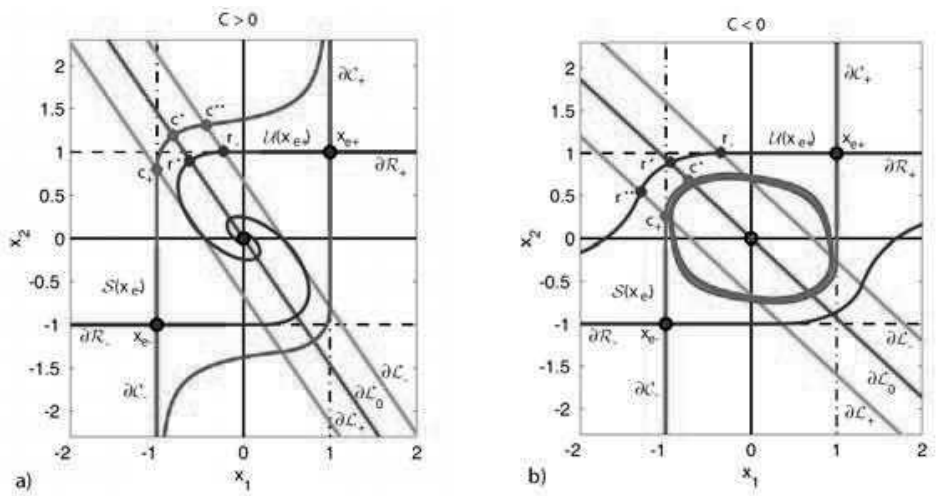

Fig. 1. The points $c^{\star}$ and $r^{\star}$ and the stable and unstable manifolds $\mathcal{S}\left(x_{e \pm}\right)$ and $\mathcal{U}\left(x_{e \pm}\right)$ for $C>0$ and $C<0$. The stable manifold can either be unbounded hyperbolically shaped (a) or end in a limit cycle (b).

This is illustrated in Fig. 1, together with the stable and unstable manifolds. A detailed description of the characteristic shape of these manifolds is given in Appendix A.

3.3. Condition for a bifurcation. Now, extending further the prolongation from $c_{+}$and $r_{-}$until the zero control straight line $\partial \mathcal{L}_{0}$ is reached, gives rise to two new intersection points $c^{*}$ and $r^{*}$.

\subsubsection{Definition of the condition on $C$.}

Definition 3.1. Let

$$
r^{*}=\Phi\left(T_{+}, r_{-}\right)=e^{(A+b f) T_{+}} r_{-}
$$

be the first intersection of the trajectory starting from $r_{-}$and $\partial \mathcal{L}_{0}$ and, similarly,

$$
c^{*}=\Phi\left(T_{-}, c_{+}\right)=e^{(A+b f) T_{-}} c_{+}
$$

( $T_{+}$and $T_{-}$are the corresponding time instants). Then $C$ is defined as follows:

$$
C=\left\|c^{*}\right\|-\left\|r^{*}\right\|=\left\|e^{(A+b f) T_{-}} c_{+}\right\|-\left\|e^{(A+b f) T_{+}} r_{-}\right\| .
$$

This condition shows whether or not the trajectory from $c_{+}$leaves $\mathcal{R}$ in backward time. If $C<0$, it does not leave $\mathcal{R}$, while it does for $C>0$. A similar argument holds for $\mathcal{C}$ and $r_{-}$. 
3.3.2. Calculation of $T_{+}$and $T_{-}$.

\section{Proposition 3.2.}

1. Distinct real poles. Let the eigenvalues of $(A+b f)$ be distinct and real. Given

$$
x_{0}=\left[\begin{array}{ll}
x_{1} & x_{2}
\end{array}\right]^{T}
$$

in $\mathcal{L} \backslash 0$, the intersection times $T_{+}$and $T_{-}$are given by

$$
\begin{aligned}
& T_{+}= \begin{cases}\gamma \quad \text { if } \gamma>0 \text { and } \alpha>0, \\
\infty \quad \text { if } \gamma \leq 0 \text { or } \alpha \leq 0,\end{cases} \\
& T_{-}= \begin{cases}\gamma & \text { if } \gamma<0 \text { and } \alpha>0, \\
\text { undefined } & \text { if } \gamma \geq 0 \text { or } \alpha \leq 0,\end{cases}
\end{aligned}
$$

where

$$
\gamma=\frac{\ln (\alpha)}{\tilde{\lambda}_{2}-\tilde{\lambda}_{1}}, \quad \alpha=\frac{f_{1} x_{1}\left(\tilde{\lambda}_{1}-\lambda_{2}\right)+f_{2} x_{2}\left(\tilde{\lambda}_{1}-\lambda_{1}\right)}{f_{1} x_{1}\left(\tilde{\lambda}_{2}-\lambda_{2}\right)+f_{2} x_{2}\left(\tilde{\lambda}_{2}-\lambda_{1}\right)} .
$$

2. Double poles. $\tilde{\lambda}_{1}=\tilde{\lambda}_{2}=\tilde{\lambda}$ leads to $\alpha=1$ and indefined $\gamma$. However, the limit value can easily be found:

$$
\begin{aligned}
& T_{+}= \begin{cases}\gamma \quad \text { if } \gamma>0, \\
\infty & \text { if } \gamma \leq 0,\end{cases} \\
& T_{-}= \begin{cases}\gamma & \text { if } \gamma<0, \\
\text { undefined } & \text { if } \gamma \geq 0,\end{cases}
\end{aligned}
$$

where

$$
\gamma=\frac{f_{1} x_{1}+f_{2} x_{2}}{f_{1} x_{1}\left(\lambda_{2}-\tilde{\lambda}\right)+f_{2} x_{2}\left(\lambda_{1}-\tilde{\lambda}\right)} .
$$

3. Complex conjugate poles. Expression (3.6) can also be used when the poles are complex. Note that the numerator and denominator of $\alpha$ are complex conjugate. Therefore, $|\alpha|=1$, the real part of $\ln (\alpha)$ is zero, and so is $\operatorname{Re}\left(\tilde{\lambda}_{2}-\tilde{\lambda}_{1}\right)$. However, the important difference is that $\ln (\alpha)$ admits multiple values, and there are infinitely many intersections, both in positive and negative times. Among the values of $\ln (\alpha)$, the first positive one and the first negative one are used for the calculation of $T_{+}$and $T_{-}$:

$$
\begin{aligned}
& T_{+}=\text {the first positive value of }\left(\frac{\ln (\alpha)}{\tilde{\lambda}_{2}-\tilde{\lambda}_{1}}\right), \\
& T_{-}=\text {the first negative value of }\left(\frac{\ln (\alpha)}{\tilde{\lambda}_{2}-\tilde{\lambda}_{1}}\right),
\end{aligned}
$$

where $\alpha$ is given by Eq. (3.6).

Proof. The proof is given in $[6,7]$. 


\section{Attraction Region as a Function of the CONDition on $C$}

In this section, results about the shape of the attraction region are formally established. Figure 2 illustrates three cases of the following theorem.

Theorem 4.1. 1. If $C>0$ (the region is bounded by hyperbolas), then:

- $\mathcal{S}\left(x_{e+}\right)$ and $\mathcal{S}\left(x_{e_{-}}\right)$are disjoint and unbounded;

- for both $\mathcal{U}\left(x_{e+}\right)$ and $\mathcal{U}\left(x_{e-}\right)$, one of the branches ends at the origin;

- the boundary of the attraction region is $\partial \mathcal{A}=\mathcal{S}\left(x_{e+}\right) \cup \mathcal{S}\left(x_{e-}\right)$.

2. If $C<0$ (the region is bounded by a limit cycle), then:

- $\mathcal{U}\left(x_{e+}\right)$ and $\mathcal{U}\left(x_{e-}\right)$ are disjoint and unbounded;

- for both $\mathcal{S}\left(x_{e+}\right)$ and $\mathcal{S}\left(x_{e-}\right)$, one of the branches ends in a limit cycle;

- the boundary of the attraction region is the unique time-reversed stable limit cycle:

$$
\partial \mathcal{A}=\lim _{t \rightarrow \infty} \Phi\left(-t, x_{0}\right) \quad \forall x_{0} \in \mathcal{U},
$$

where the boundary of $\mathcal{U}$ is $\partial \mathcal{U}=\mathcal{U}\left(x_{e+}\right) \cup \mathcal{U}\left(x_{e-}\right)$.

3. If $C=0$ (the region is bounded by two heteroclinic connections), then:

- one of the branches of $\mathcal{U}\left(x_{e+}\right)$ is bounded and coincides with that of $\mathcal{S}\left(x_{e-}\right)$

- one of the branches of $\mathcal{U}\left(x_{e_{-}}\right)$is bounded and coincides with that of $\mathcal{S}\left(x_{e+}\right)$

- the boundary of the attraction region is a double heteroclinic connection, $\partial \mathcal{A}=\left(\mathcal{U}\left(x_{e+}\right) \cap \mathcal{S}\left(x_{e-}\right)\right) \cup\left(\mathcal{U}\left(x_{e-}\right) \cap \mathcal{S}\left(x_{e+}\right)\right)$.

Proof. In what follows, we consider the region

$$
\mathcal{D}=\mathcal{C} \cap \mathcal{R}=\left\{x \in \mathbb{R}^{2}:\left|x_{1}\right|<1 \text { and }\left|x_{2}\right|<1\right\} .
$$

Case 1: $C>0$. Since $\mathcal{S}\left(x_{e \pm}\right) \subset \overline{\mathcal{C}}$ by Lemma A.1, the connected set $\mathcal{S}_{0} \subset \mathcal{C}$ is defined as the set bounded by $\partial \mathcal{S}_{0}=\mathcal{S}\left(x_{e+}\right) \cup \mathcal{S}\left(x_{e-}\right)$ but not containing the boundary, i.e., $\partial \mathcal{S}_{0} \not \subset \mathcal{S}_{0}$. Since $\partial \mathcal{S}_{0}$ are trajectories, we obtain from the existence and uniqueness theorem that a trajectory starting from $\mathcal{S}_{0}$ must remain in this set. Consider the bounded closed set $\overline{\mathcal{B}}$, which is a part of $\overline{\mathcal{S}_{0}}$, delimited by $-1 \leq x_{2} \leq 1$, i.e., $\overline{\mathcal{B}}=\overline{\mathcal{S}_{0}} \cap \overline{\mathcal{D}}$ with $\mathcal{B}=\overline{\mathcal{B}} \backslash \partial \mathcal{B}$. Now, we consider the stable part of system (2.3), for which

$$
\begin{array}{ll}
\dot{x}_{2}=\lambda_{2}\left(x_{2}+u\right) \geq 0 & \text { for } x_{2} \leq-1 \text { and } u \in \mathcal{U}_{a}=[-1,1], \\
\dot{x}_{2}=\lambda_{2}\left(x_{2}+u\right) \leq 0 & \text { for } x_{2} \geq 1 \text { and } u \in \mathcal{U}_{a}=[-1,1] .
\end{array}
$$

i.e., the vector fields always point inward $\overline{\mathcal{B}}$. Then, since there is no equilibrium point in $\overline{\mathcal{S}_{0}} \backslash \overline{\mathcal{B}}$, it follows that all trajectories starting from $\overline{\mathcal{S}_{0}} \backslash \overline{\mathcal{B}}$ 

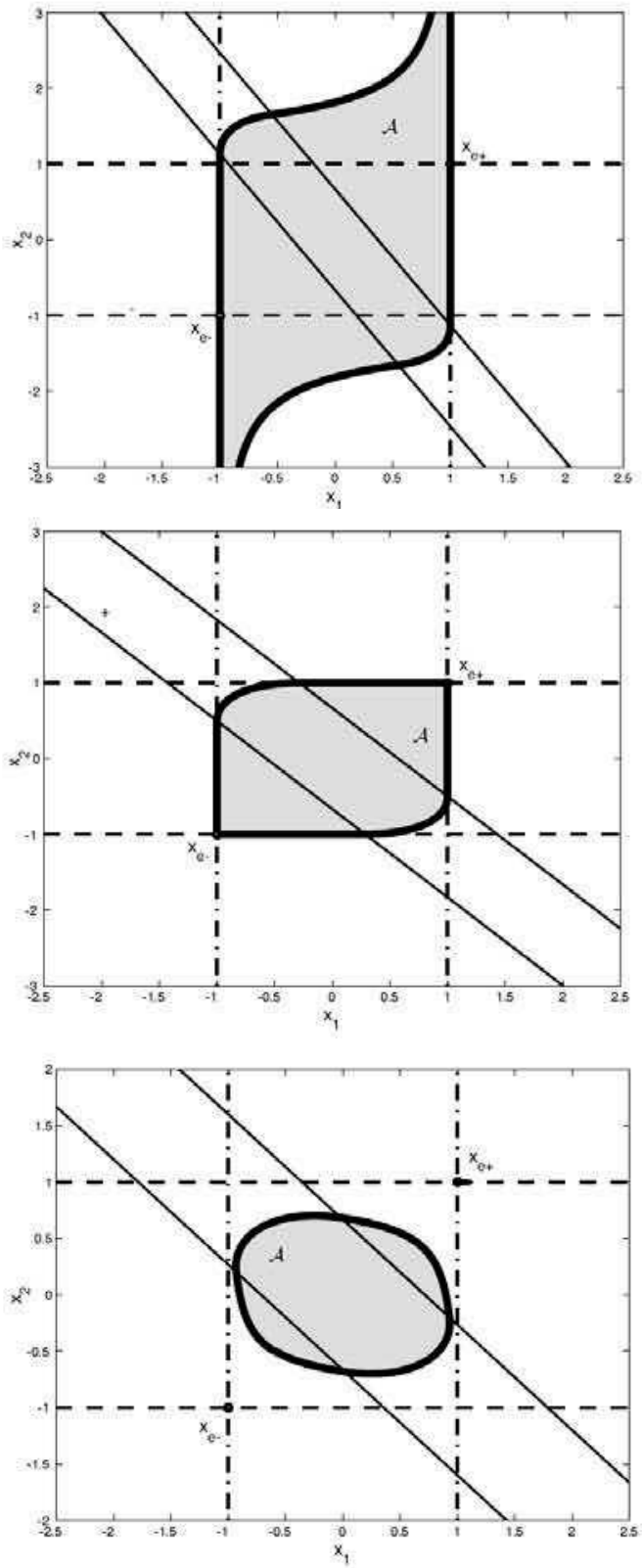

Fig. 2. A bifurcation of the attraction region happens when $C=0$ ( $C>0$ (top), $C=0$ (middle), and $C<0$ ). 
enter and remain in $\overline{\mathcal{B}}$. Thus, $\overline{\mathcal{B}}$ is a closed bounded invariant set, which contains three equilibrium points $x_{e+}, x_{e-}$, and the origin $x_{e 0}$.

According to the Poincaré-Bendixson theorem [13] and Lemma B.1 (see Appendix B), all trajectories starting from $\overline{\mathcal{B}}$ will converge either to $x_{e+}$, $x_{e-}, x_{e 0}$ or to a limit cycle encircling $x_{e 0}$. Since $x_{e \pm} \in \partial \mathcal{B} \cap \partial \mathcal{S}_{0}$, only the trajectories starting from the part of the stable manifolds $\partial \mathcal{B} \cap \partial \mathcal{S}_{0}$ converge to $x_{e \pm}$. Thus, all other points in $\overline{\mathcal{B}}$, denoted by $\overline{\mathcal{B}} \backslash \partial \mathcal{S}_{0}$, converge to the origin $x_{e 0}$ or to a limit cycle encircling $x_{e 0}$.

Next, it is shown that there exists no limit cycle, which implies that $\mathcal{S}_{0}$ is the attraction region $\mathcal{A}$, where $\partial \mathcal{A}=\partial \mathcal{S}_{0}=\mathcal{S}\left(x_{e+}\right) \cup \mathcal{S}\left(x_{e-}\right)$. Moreover, one of the branches of $\mathcal{U}\left(x_{e \pm}\right)$ converges to $x_{e 0}$. The proof is divided into two cases.

Case $\lambda_{1}+\lambda_{2} \leq 0$. According to the proof of the Bendixson theorem [13], if there exists a limit cycle, whose closed orbit is denoted by $\partial \mathcal{L C}$ and the region encircled by it is denoted by $\mathcal{L C}$, then the integral over the area $\mathcal{L C}$

$$
\iint_{\mathcal{L} \mathcal{C}} \nabla \cdot \dot{x} d x_{1} d x_{2}=0,
$$

where $\nabla \cdot \dot{x}$ is the divergence of the vector field of the closed-loop system (2.5)

$$
\begin{aligned}
\nabla \cdot \dot{x} & =\frac{\partial \dot{x}_{1}}{\partial x_{1}}+\frac{\partial \dot{x}_{2}}{\partial x_{2}} \\
& = \begin{cases}D_{L}:=\lambda_{1}\left(1+f_{1}\right)+\lambda_{2}\left(1+f_{2}\right) & \text { if } x \in \mathcal{L}, \\
D_{S}:=\lambda_{1}+\lambda_{2} & \text { if } x \in \mathbb{R}^{2} \backslash \mathcal{L} .\end{cases}
\end{aligned}
$$

Since the system in $\mathcal{L}$ is a Hurwitz system, Condition (2.6) holds and $D_{L}<$ 0 . Since only the case $\lambda_{1}+\lambda_{2} \leq 0$ is investigated, it follows that $D_{S} \leq 0$. Moreover, the origin $x_{e 0}$ lies in the stable region $\mathcal{L}$, and, therefore, the integral

$$
\iint_{S} \nabla \cdot \dot{x} d x_{1} d x_{2}<0
$$

is always negative over any area $S \subset \mathbb{R}^{2}$ containing the origin. Now, assume that there exists a limit cycle. Then, since the limit cycle has to encircle the stable node located at the origin and since integral (4.2) does not vanish, a limit cycle cannot exist.

Case: $\lambda_{1}+\lambda_{2}>0$. Consider $f \dot{x}$ for all $x \in \mathcal{L}_{+}\left(\right.$or $x \in \mathcal{L}_{-}$), and define the points

$$
\begin{aligned}
p_{0} \in \mathcal{L}_{+}, & \text {for which } f \dot{x}\left(p_{0}\right)=0, \\
-p_{0} \in \mathcal{L}_{-}, & \text {for which } f \dot{x}\left(-p_{0}\right)=0,
\end{aligned}
$$

which are shown in Fig. 3. Two branches on $\partial \mathcal{L}_{+}$(respectively, on $\left.\partial \mathcal{L}_{-}\right)$ issue from the point $p_{0}$ (respectively, $-p_{0}$ ). For one of them $f \dot{x}>0$, and for the other $f \dot{x}<0$. 


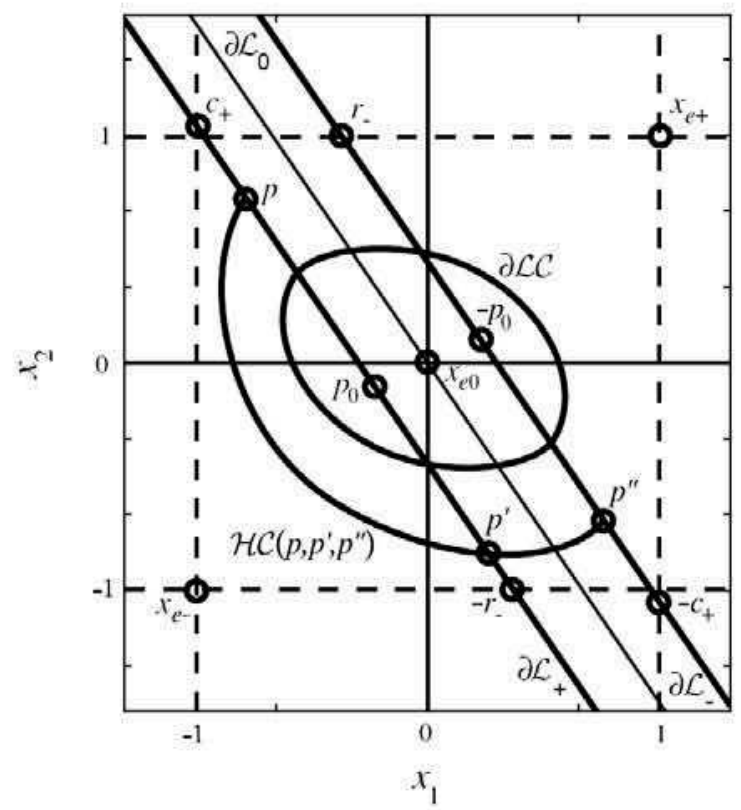

Fig. 3. The illustration of the points $p_{0}$ and $-p_{0}$. The qualitative characterization of a limit cycle and illustration of a half-cycle.

Lemma B.3 (see Appendix B) characterizes qualitatively the existence and shape of one or more limit cycles as a function of the sign of the controller parameter $f_{2}$. Assume that, according to Lemma B.3, there exists a limit cycle referred to as $\partial \mathcal{L C}$ (its interior is denoted by $\mathcal{L C}$ ). Then, $\partial \mathcal{L C}$ encircles the points $x_{e_{0}}, p_{0}$, and $-p_{0}$ (see Fig. 3). Therefore, there exist trajectories encircling $p_{0} \in \mathcal{L C},-p_{0} \in \mathcal{L C}$, and $x_{e 0} \in \mathcal{L C}$ which converge to $\partial \mathcal{L C}$, either in forward time, whenever $\partial \mathcal{L C}$ is stable, or in reversed time, whenever $\partial \mathcal{L C}$ is unstable.

Definition 4.2. A half-cycle is defined according to Fig. 3. It is denoted by $\mathcal{H C}\left(p, p^{\prime}, p^{\prime \prime}\right)$ and starts from $p \in \overline{p_{0} c_{+}} \backslash p_{0} \subset \partial \mathcal{L}_{+}$, crosses $p^{\prime} \in \overline{p_{0}-r_{-}} \backslash$ $p_{0} \subset \partial \mathcal{L}_{+}$, and ends at $p^{\prime \prime} \in \overline{-p_{0}-c_{+}} \backslash-p_{0} \subset \partial \mathcal{L}_{-}$.

By symmetry, $\partial \mathcal{L C}=\mathcal{H C}\left(p, p^{\prime}, p^{\prime \prime}\right) \cup-\mathcal{H C}\left(p, p^{\prime}, p^{\prime \prime}\right)$. Now, assume that there exists at least one limit cycle in $\mathcal{B}$. Denote the closed curve of the largest limit cycle by $\partial \mathcal{L C}_{\max }$ (its interior is $\mathcal{L C}_{\max }$ ). Then, all trajectories starting from $\mathcal{B} \backslash \mathcal{L} \mathcal{C}_{\max }$ encircle $\partial \mathcal{L C}$ max and converge to $\partial \mathcal{L C}_{\max }$ as $t \rightarrow \infty$. According to Lemma B.3, the limit cycle $\partial \mathcal{L C}_{\max }$ encircles $p_{0},-p_{0}$, and $x_{e 0}$. Using Definition 4.2, two arbitrary half-cycles $\mathcal{H C}_{1}\left(p_{1}, p_{1}^{\prime}, p_{1}^{\prime \prime}\right)$ and $\mathcal{H C}_{2}\left(p_{2}, p_{2}^{\prime}, p_{2}^{\prime \prime}\right)$ located in $\mathcal{B} \backslash \mathcal{L} \mathcal{C}_{\text {max }}$ are defined. If $\partial \mathcal{L C}_{\text {max }}$ exists, then the 
distance between the two half-cycles has to shorten:

$$
\left\|p_{1}-p_{2}\right\|>\left\|p_{1}^{\prime \prime}-p_{2}^{\prime \prime}\right\| \text {. }
$$

It follows from Lemmas C.5 and C. 2 that, since $\lambda_{1}+\lambda_{2}>0$ is considered,

$$
\left\|p_{1}^{\prime}-p_{2}^{\prime}\right\|<\left\|p_{1}^{\prime \prime}-p_{2}^{\prime \prime}\right\|, \quad\left\|p_{1}-p_{2}\right\|<\left\|p_{1}^{\prime}-p_{2}^{\prime}\right\| .
$$

Therefore,

$$
\left\|p_{1}-p_{2}\right\|<\left\|p_{1}-p_{2}\right\|<\left\|p_{1}^{\prime \prime}-p_{2}^{\prime \prime}\right\|
$$

holds in the whole region $\mathcal{B}$, which is a contradiction to (4.3). Therefore, no limit cycle can exist within $\mathcal{B}$.

Case 2: $C<0$. Lemma A.1 shows that $\mathcal{U}\left(x_{e \pm}\right) \subset \overline{\mathcal{R}}$. Hence, it is possible to define a connected set $\mathcal{U}_{0} \subset \mathcal{R}$ with boundaries $\partial \mathcal{U}_{0}=\mathcal{U}\left(x_{e+}\right) \cup$ $\mathcal{U}\left(x_{e-}\right)$ and $\partial \mathcal{U}_{0} \not \subset \mathcal{U}_{0}$. Since $\partial \mathcal{U}_{0}$ are trajectories, we have, by invoking the existence and uniqueness theorem, that a trajectory starting within $\mathcal{U}_{0}$ must remain in this set. Consider the bounded and closed set $\overline{\mathcal{B}}=\overline{\mathcal{U}_{0}} \cap \overline{\mathcal{D}}$, where $\mathcal{B}=\overline{\mathcal{B}} \backslash \partial \mathcal{B}$. Similarly to the case $C>0$, and by considering the unstable part of system $(2.3)$

$$
\begin{gathered}
\dot{x}_{1}=\lambda_{1}\left(x_{1}+u\right) \leq 0 \quad \text { for } x_{1} \leq-1 \text { and } u \in \mathcal{U}_{a}=[-1,1], \\
\dot{x}_{1}=\lambda_{1}\left(x_{1}+u\right) \geq 0 \quad \text { for } x_{1} \geq 1 \text { and } u \in \mathcal{U}_{a}=[-1,1],
\end{gathered}
$$

we obtain that all trajectories starting from $\mathcal{U}_{0} \backslash \mathcal{B}$ enter and remain within $\mathcal{B}$. Moreover, they converge either to $x_{e 0}$ or to a limit cycle encircling $x_{e 0}$. The equilibrium point $x_{e 0}$ is unstable and, therefore, at least one limit cycle exists. It remains to show that this cycle is unique.

Note that $C<0$ implies $\lambda_{1}+\lambda_{2}>0$. Indeed, Case 1 shows that a limit cycle cannot exist if $\lambda_{1}+\lambda_{2} \leq 0$. This argument holds as well for the case $C<0$. Since there always exists a limit cycle if $C<0$, the condition $\lambda_{1}+\lambda_{2}>0$ must be satisfied in this case.

Let us assume that there exist two arbitrary limit cycles $\partial \mathcal{L C}_{1}$ and $\partial \mathcal{L C}_{2}$ which, according to Lemma B.3, are located in $\mathcal{B}$ and encircle $p_{0},-p_{0}$, and $x_{e 0}$. By means of Definition 4.2, consider both corresponding half-cycles $\mathcal{H C}_{1}\left(p_{1}, p_{1}^{\prime}, p_{1}^{\prime \prime}\right) \subset \partial \mathcal{L C}_{1}$ and $\mathcal{H C}_{2}\left(p_{2}, p_{2}^{\prime}, p_{2}^{\prime \prime}\right) \subset \partial \mathcal{L C}_{2}$, where $p_{1}=-p_{1}^{\prime \prime}$ and $p_{2}=-p_{2}^{\prime \prime}$. This leads to

$$
\left\|p_{1}-p_{2}\right\|=\left\|p_{1}^{\prime \prime}-p_{2}^{\prime \prime}\right\|
$$

It follows from Lemma C.5 and C.2 that, since $\lambda_{1}+\lambda_{2}>0$,

$$
\left\|p_{1}^{\prime}-p_{2}^{\prime}\right\|<\left\|p_{1}^{\prime \prime}-p_{2}^{\prime \prime}\right\|, \quad\left\|p_{1}-p_{2}\right\|<\left\|p_{1}^{\prime}-p_{2}^{\prime}\right\| .
$$

Therefore,

$$
\left\|p_{1}-p_{2}\right\|<\left\|p_{1}-p_{2}\right\|<\left\|p_{1}^{\prime \prime}-p_{2}^{\prime \prime}\right\|
$$

holds in the whole region $\mathcal{B}$, which is a contradiction to (4.4). Therefore, there exists only one limit cycle in $\mathcal{B}$. Moreover, all trajectories starting from $\mathcal{U}_{0} \backslash x_{e 0}$ converge to it in reversed time. Thus, since $\mathcal{S}\left(x_{e \pm}\right) \subset \mathcal{U}_{0}$, 
one branch of each stable manifold converges to the limit cycle. Hence, the limit cycle is the boundary of the attraction region $\mathcal{A}$.

Case 3: $C=0$. The sets $\mathcal{S}_{0}=\mathcal{B}$ are defined as bounded by the stable or unstable manifolds $\partial \mathcal{S}_{0}=\partial \mathcal{B}=\mathcal{S}\left(x_{e+}\right) \cup \mathcal{S}\left(x_{e-}\right)=\mathcal{U}\left(x_{e+}\right) \cup \mathcal{U}\left(x_{e-}\right)$. Hence $\partial \mathcal{S}_{0}$ is bounded by trajectories and, therefore, no trajectory starting from $\mathbb{R}^{2} \backslash \mathcal{S}_{0}$ can enter $\mathcal{S}_{0}$. Moreover, all trajectories starting from $\mathcal{S}_{0}$ remain in it. Following to a similar argument as before, there exists no limit cycle in $\mathcal{S}_{0}$, which implies that $\mathcal{S}_{0}$ is the attraction region $\mathcal{A}$, where $\partial \mathcal{A}=\mathcal{S}\left(x_{e+}\right) \cup \mathcal{S}\left(x_{e-}\right)=\mathcal{U}\left(x_{e+}\right) \cup \mathcal{U}\left(x_{e-}\right)$.

\section{Conclusion}

This paper analyzes the bifurcation of the attraction region for linear planar systems having one stable and one unstable pole. It is shown that a bifurcation occurs when the unstable pole is on the verge of becoming faster than the stable one. An analytical condition is provided for which the attraction region changes from an unbounded hyperbolic region to a bounded limit cycle. The results presented rely mainly on two different complementary methodologies:

- the fundamental theorems of Poincaré and Bendixson that give results on the existence of limit cycles for second-order systems;

- some results from contraction analysis for second-order systems (see Appendix C), where one part is provided by [8] and the other part is a new contribution.

\section{Appendix A. Qualitative Description \\ OF THE STABLE AND UNSTABLE MANIFOLDS \\ OF THE SADDLE EQUILIBRIUM POINTS}

For the description of the stable and unstable manifolds, the following lemmas and definition are required.

Lemma A.1. Consider system (2.5). The stable manifold $\mathcal{S}\left(x_{e \pm}\right)$ is located in the closure of the null controllable region

$$
\mathcal{S}\left(x_{e \pm}\right) \subset \overline{\mathcal{C}}
$$

and the unstable manifold $\mathcal{U}\left(x_{e \pm}\right)$ is located in the closure of the null reachable region

$$
\mathcal{U}\left(x_{e \pm}\right) \subset \overline{\mathcal{R}} .
$$

Proof. Since for states $x$ located outside $\overline{\mathcal{C}}$, the unstable part of system (2.3) satisfies the conditions

$$
\begin{gathered}
\dot{x}_{1}=\lambda_{1}\left(x_{1}+u\right)>0 \quad \text { for } x_{1}>1 \text { and } u \in \mathcal{U}_{a}=[-1,1], \\
\dot{x}_{1}=\lambda_{1}\left(x_{1}+u\right)<0 \quad \text { for } x_{1}<-1 \text { and } u \in \mathcal{U}_{a}=[-1,1],
\end{gathered}
$$


there exists no admissible control $u \in \mathcal{U}_{a}$, which could steer the system to $x_{e+}$ or $x_{e-}$. Thus, the stable manifolds $\mathcal{S}\left(x_{e \pm}\right)$ are located in $\overline{\mathcal{C}}$. The proof of the fact that $\mathcal{U}\left(x_{e \pm}\right) \subset \overline{\mathcal{R}}$ is similar by considering the time-reversed system for (2.3).

Lemma A.2. Consider a planar linear system with an equilibrium point at the origin. Each trajectory crossing a ray $\mathrm{R}(r)$, defined by

$$
\mathrm{R}(r)=\left\{x \in \mathbb{R}^{2}: x=\mu r, \mu \geq 0, r \in \mathbb{R}^{2}\right\},
$$

can cross again the same ray $\mathrm{R}(r)$ if and only if it encircles the equilibrium point. Thus, each trajectory crosses the ray in the same direction.

Proof. Consider both an arbitrary second-order autonomous linear system defined by $\dot{x}=A x$ and a ray $\mathrm{R}(r)$ with

$$
r=\left[\begin{array}{ll}
a & b
\end{array}\right]^{T} .
$$

The direction of the trajectory crossing the ray $\mathrm{R}(r)$ is determined by the sign of

$$
r^{\prime T} \dot{x}=r^{\prime T} A x=r^{\prime T} A r \mu .
$$

Since $\mu \geq 0$ and $r^{\prime T} A r$ is a scalar, this expression either has the same sign or is zero. If $r^{\prime T} \dot{x}=0$, then the trajectory either reaches $\mathrm{R}(r)$ or stays in $\mathrm{R}(r)$ without crossing it.

Since (2.5) is Lipschitz, it has a unique solution for any initial condition (the existence and uniqueness theorem for nonlinear systems [10]). Thus, trajectories cannot cross one another.

Definition A.3. The part of a trajectory starting from the point $a \in \mathbb{R}^{2}$ and ending at the point $b \in \mathbb{R}^{2}$ is denoted by $\operatorname{Trj}(a, b)$.

The qualitative description of the stable and unstable manifolds concerns the analysis of the time evolution of these manifolds. For the stable manifolds, the evolution in the reversed time is considered.

First, consider the evolution of the manifolds inside the saturated regions. The state translation,

$$
x=\bar{x}-A^{-1} b \bar{u}, \quad \text { where } \bar{u}=1 \text { or } \bar{u}=-1,
$$

changes the system dynamics to $\dot{\bar{x}}=A \bar{x}$. Since $A$ is diagonal, the stable and unstable manifolds are given by

$$
\overline{\mathcal{S}}(0)=\left\{\bar{x} \in \mathbb{R}^{2}: \bar{x}_{1}=0\right\}, \quad \overline{\mathcal{U}}(0)=\left\{\bar{x} \in \mathbb{R}^{2}: \bar{x}_{2}=0\right\},
$$

respectively. But since the whole state space of the closed-loop system (2.5) is considered, the manifolds $\overline{\mathcal{S}}(0)$ and $\overline{\mathcal{U}}(0)$ are translated by $x_{e+}$ in the positive saturated region and by $x_{e-}$ in the negative saturated region. Therefore, from the saddle points $x_{e \pm}$, the branches of the manifolds $\mathcal{S}\left(x_{e-}\right)$ and $\mathcal{U}\left(x_{e+}\right)$ extend along $\partial \mathcal{R}$ and $\partial \mathcal{C}$ until they reach the linear region. The 
two points, where the manifolds $\mathcal{S}\left(x_{e-}\right)$ and $\mathcal{U}\left(x_{e+}\right)$ intersect the boundaries of the linear region, are given by (3.1) and (3.2). The following intersection points are also introduced:

$$
\begin{aligned}
c_{0}=\partial \mathcal{C}_{-} \cap \partial \mathcal{L}_{0}, & c_{-}=\partial \mathcal{C}_{-} \cap \partial \mathcal{L}_{-}, \\
r_{0}=\partial \mathcal{R}_{-} \cap \partial \mathcal{L}_{0}, & r_{+}=\partial \mathcal{R}_{-} \cap \partial \mathcal{L}_{+} .
\end{aligned}
$$

Since the remaining part of the manifolds evolves in the linear region $\mathcal{L}$, three different cases appear depending on the sign of $f_{2}$ ( $f_{1}$ is negative).

1. $f_{2}=0$. There is no intersection of the stable manifold $\mathcal{S}\left(x_{e-}\right)$ with $\mathcal{L}$ since $c_{+}$is not defined for $f_{2}=0 . \mathcal{S}\left(x_{e-}\right)$ stays in $\overline{\mathcal{L}_{+}}$and hence $\mathcal{S}\left(x_{e-}\right)=\left\{x \in \mathbb{R}^{2}: x_{1}=-1\right\}$ and $\mathcal{S}\left(x_{e+}\right)=\left\{x \in \mathbb{R}^{2}: x_{1}=1\right\}$. One branch of $\mathcal{U}\left(x_{e+}\right)$ stays inside the null controllable region $\mathcal{C}$ and the other branch is located in the negative saturated region $\overline{\mathcal{L}_{-}}$.

2. $f_{2}<0$. By Lemma A.1, the stable manifold $\mathcal{S}\left(x_{e-}\right)$ cannot cross the segment $\overline{c_{+} c_{0}}$. By Lemma A.2, it cannot cross the ray $\mathrm{R}\left(c_{+}\right)$. Thus, it has to cross the segment $\overline{x_{e 0} c_{0}} \backslash x_{e 0}$ of $\partial \mathcal{L}_{0}$, where the intersection is denoted by $c^{\star}$ (see Fig. 1). Similarly, using Lemmas A.1 and A.2, the unstable manifold $\mathcal{U}\left(x_{e+}\right)$ cannot cross the segment $\overline{r_{-} r_{0}}$ and the ray $\mathrm{R}\left(r_{-}\right)$, respectively. Thus, it has to cross the segment $\overline{x_{e 0} r_{0}}$ of $\partial \mathcal{L}_{0}$, where the intersection is denoted by $r^{\star}$. Since the intersection of both potentially crossed segments is not empty, three cases are possible.

(a) $\left\|c^{\star}\right\|>\left\|r^{\star}\right\|$. The stable manifold $\mathcal{S}\left(x_{e-}\right)$ cannot cross:

(i) the segment $\overline{c_{0} c_{-}}$(Lemma A.1),

(ii) the ray $\mathrm{R}\left(c^{\star}\right)$ (Lemma $\mathrm{A} .2$ ), and

(iii) the part of the trajectory $\operatorname{Trj}\left(r_{-}, r^{\star}\right)$ (the existence and uniqueness theorem).

Thus, it must cross the segment $\overline{r_{-} c_{-}} \backslash r_{-}$of $\partial \mathcal{L}_{-}$, where the intersection is denoted by $c^{\star \star}$ (see Fig. 1). At $c^{\star \star}$, the stable manifold evolves hyperbolically inside the negative saturated region according to the dynamics of the linear system with the state translation (A.2). Since Lemma A.1 states that $\mathcal{U}\left(x_{e+}\right) \subset \overline{\mathcal{R}}$, the unstable manifold $\mathcal{U}\left(x_{e+}\right)$ remains in the region bounded by both stable manifolds $\mathcal{S}\left(x_{e \pm}\right)$. Moreover, it converges to $x_{e 0}$.

(b) $\left\|c^{\star}\right\|<\left\|r^{\star}\right\|$. The unstable manifold $\mathcal{U}\left(x_{e+}\right)$ cannot cross:

(i) the segment $\overline{r_{0} r_{+}}$(Lemma A.1),

(ii) the ray $\mathrm{R}\left(r^{\star}\right)$ (Lemma A.2), and

(iii) the part of the trajectory $\operatorname{Trj}\left(c_{+}, c^{\star}\right)$ (the existence and uniqueness theorem).

Thus, it has to cross the segment $\overline{c_{+} r_{+}} \backslash c_{+}$of $\partial \mathcal{L}_{+}$, where the intersection is denoted by $r^{\star \star}$. At $r^{\star \star}$, the unstable manifold evolves hyperbolically inside the negative saturated region according to the dynamics of the linear system with the state translation (A.2). Since Lemma A.1 states that $\mathcal{S}\left(x_{e-}\right) \subset \overline{\mathcal{C}}$, the stable manifold 
$\mathcal{S}\left(x_{e-}\right)$ remains in the region bounded by both unstable manifolds $\mathcal{U}\left(x_{e \pm}\right)$. Moreover, it converges to a limit cycle (see Fig. 1).

(c) $\left\|c^{\star}\right\|=\left\|r^{\star}\right\|$. In this case, the stable manifold $\mathcal{S}\left(x_{e-}\right)$ converges to $x_{e+}$ in the reversed time and the unstable manifold $\mathcal{U}\left(x_{e+}\right)$ converges to $x_{e-}$ in the forward time. As was proved in Sec. 4, each trajectory evolving in the region bounded by $\mathcal{S}\left(x_{e-}\right)=\mathcal{U}\left(x_{e+}\right)$ and $\mathcal{S}\left(x_{e+}\right)=\mathcal{U}\left(x_{e-}\right)$ converges to the origin $x_{e 0}$.

3. $f_{2}>0$. Reformulating the second Hurwitz condition (2.7), we obtain $\frac{1+f_{1}}{f_{2}}<-1$. Thus, the point $c_{+}$lies always outside the null reachable region $\mathcal{R}$ in the state space $\overline{\mathcal{R}_{-}}=\left\{x \in \mathbb{R}^{2}: x_{2}<-1\right\}$. In $\overline{\mathcal{R}_{-}}$, the vector field $\dot{x}$ points always to $\mathcal{R}$ since

$$
\dot{x}_{2}=\lambda_{2}\left(x_{2}+u\right)>0 \quad \text { for } x_{2}<-1 \text { and } u \in \mathcal{U}_{a}=[-1,1] .
$$

Moreover, Lemma A.1 states that $\mathcal{S}\left(x_{e-}\right) \subset \overline{\mathcal{C}}$. Therefore, the stable manifold $\mathcal{S}\left(x_{e_{-}}\right)$inevitably crosses $\partial \mathcal{L}_{0}$ and $\partial \mathcal{L}_{-}$, where the intersection points are denoted by $c^{\star} \in \overline{\mathcal{R}_{-}}$and $c^{\star \star} \in \overline{\mathcal{R}_{-}}$, respectively. At $c^{\star \star}$, the stable manifold evolves hyperbolically inside the negative saturated region according to the dynamics of the linear system with the state translation (A.2). Since Lemma A.1 states that $\mathcal{U}\left(x_{e+}\right) \subset \overline{\mathcal{R}}$, the unstable manifold $\mathcal{U}\left(x_{e+}\right)$ crosses or reaches $\partial \mathcal{L}_{0}$ inside $\overline{\mathcal{R}}$, where the intersection is denoted by $r^{\star}$. Thus, the condition $\left\|c^{\star}\right\|>\left\|r^{\star}\right\|$ always holds.

\section{Appendix B. Lemmas AND Definitions USED IN THE PROOF OF THEOREM 4.1}

Consider the region

$$
\mathcal{D}=\mathcal{C} \cap \mathcal{R}=\left\{x \in \mathbb{R}^{2}:\left|x_{1}\right|<1 \text { and }\left|x_{2}\right|<1\right\} .
$$

Lemma B.1. Consider system (2.3) with the admissible input $u \in \mathcal{U}_{a}=$ $[-1,1]$. A limit cycle can occur only in the region $\mathcal{D}$ around the equilibrium point $x_{e 0} \in \mathcal{D}$.

Proof. According to Theorem 2.1, system (2.3) with the input $u \in \mathcal{U}_{a}$ has three equilibrium points, $x_{e 0}, x_{e+}$, and $x_{e-}$. Since, by Appendix A, the stable and unstable manifolds of $x_{e \pm}$ possess always at least one unbounded branch, no trajectory can encircle $x_{e \pm}$. Thus, by the Poincaré theorem [13], if a limit cycle exists, it has to encircle the stable equilibrium point $x_{e 0}$.

In the region $\mathbb{R}^{2} \backslash \mathcal{C}=\left\{x \in \mathbb{R}^{2}:\left|x_{1}\right| \geq 1\right\}$, the unstable part of system (2.3) gives:

$$
\begin{gathered}
\dot{x}_{1}=\lambda_{1}\left(x_{1}+u\right) \leq 0 \quad \text { for } x_{1} \leq-1 \text { and } u \in \mathcal{U}_{a}=[-1,1], \\
\dot{x}_{1}=\lambda_{1}\left(x_{1}+u\right) \geq 0 \quad \text { for } x_{1} \geq 1 \text { and } u \in \mathcal{U}_{a}=[-1,1] .
\end{gathered}
$$


Therefore, a trajectory leaving the region $\mathcal{C}$ cannot reenter it. Similarly, in the region $\mathbb{R}^{2} \backslash \mathcal{R}=\left\{x \in \mathbb{R}^{2}:\left|x_{2}\right| \geq 1\right\}$, the stable part of system (2.3) gives:

$$
\begin{gathered}
\dot{x}_{2}=\lambda_{2}\left(x_{2}+u\right) \geq 0 \quad \text { for } x_{2} \leq-1 \text { and } u \in \mathcal{U}_{a}=[-1,1], \\
\dot{x}_{2}=\lambda_{2}\left(x_{2}+u\right) \leq 0 \quad \text { for } x_{2} \geq 1 \text { and } u \in \mathcal{U}_{a}=[-1,1] .
\end{gathered}
$$

It follows that a trajectory entering the region $\mathcal{R}$ stays in it. Therefore, there exists no limit cycle for which a part of the trajectory is located in $\mathbb{R}^{2} \backslash \mathcal{D}$.

The region where the limit cycle is located in $\mathcal{D}$ is now described. Since the closed-loop system (2.5) is stable in the linear region, there cannot exist any limit cycle in it. A part of the limit cycle has to pass through the positive and negative saturated regions crossing $\partial \mathcal{L}_{+}$and $\partial \mathcal{L}_{-}$. Since system $(2.5)$ is symmetric, only $\partial \mathcal{L}_{+}$is considered. To determine whether a trajectory exists or enters the linear region, the vector field $\dot{x}(x)$ of system (2.3) for all $x \in \partial \mathcal{L}_{+}$and $u=1$ is studied.

- If $f \dot{x}\left(x \in \partial \mathcal{L}_{+}\right)>0$, then the trajectory exits the linear region $\mathcal{L}$.

- If $f \dot{x}\left(x \in \partial \mathcal{L}_{+}\right)<0$, then the trajectory enters the linear region $\mathcal{L}$.

- If $f \dot{x}\left(x \in \partial \mathcal{L}_{+}\right)=0$, then the vector field is parallel to $\partial \mathcal{L}_{+}$and the trajectory stays in $\partial \mathcal{L}_{+}$.

The inner product

$$
f \dot{x}\left(x \in \partial \mathcal{L}_{+}\right)=f_{1} \lambda_{1}\left(x_{1}+1\right)+f_{2} \lambda_{2}\left(x_{2}+1\right)
$$

can be expressed as a function of only one state variable, e.g., $x_{2}$, if $x_{1}$ is substituted from the equation $f x=f_{1} x_{1}+f_{2} x_{2}=1$ defining $\partial \mathcal{L}_{+}$:

$$
f \dot{x}\left(x \in \partial \mathcal{L}_{+}\right)=f_{2}\left(\lambda_{2}-\lambda_{1}\right)\left(1+x_{2}\right)+\lambda_{1}\left(1+f_{1}+f_{2}\right) .
$$

This equation is obviously linearly dependent on $x_{2}$, and, therefore, there exists only one point for which it is zero, i.e.,

$$
p_{0} \in \mathcal{L}_{+} \text {for which } f \dot{x}\left(p_{0}\right)=0 .
$$

Starting from the point $p_{0}$, there exist two branches on $\partial \mathcal{L}_{+}$. For one of them $f \dot{x}>0$ and for the other $f \dot{x}<0$.

Lemma B.2. Consider the points $p_{0}, c_{+}$, and $-r_{-}$and the closed-loop system (2.5). The point $p_{0}$ is always located on the segment bounded by $c_{+}$ and $-r_{-}, p_{0} \in \overline{c_{+}-r_{-}} \subset \partial \mathcal{L}_{+}$. Furthermore, for all $x \in \overline{p_{0} c_{+}} \backslash p_{0}$, the trajectory of system $(2.5)$ exits the linear region $\mathcal{L}$, since $f \dot{x}(x)>0$, and enters $\mathcal{L}$ for all $x \in \overline{p_{0}-r_{-}} \backslash p_{0}$, since $f \dot{x}(x)<0$. 
Proof. The points $p_{0}, c_{+}$, and $-r_{-}$are elements of $\partial \mathcal{L}_{+}$. Since the second Hurwitz condition $1+f_{1}+f_{2}<0$ is satisfied, the following relations hold:

$$
\begin{gathered}
f \dot{x}\left(p_{0}\right)=0, \\
f \dot{x}\left(c_{+}\right)=\lambda_{2}\left(1+f_{1}+f_{2}\right)>0, \\
f \dot{x}\left(-r_{-}\right)=\lambda_{1}\left(1+f_{1}+f_{2}\right)<0 .
\end{gathered}
$$

This means that the point $p_{0}$ is an element of $\overline{c_{+}-r_{-}} \subset \partial \mathcal{L}_{+}$. Similarly, $f \dot{x}(x)>0$ for all $x \in \overline{p_{0} c_{+}} \backslash p_{0}$ and $f \dot{x}(x)<0$ for all $x \in \overline{p_{0}-r_{-}} \backslash p_{0}$.

Lemma B.3. Consider closed-loop system (2.5) and the controller parameter $f_{2} \in \mathbb{R}$. Then

- $f_{2} \geq 0$ implies that there are no limit cycles;

- $f_{2}<0$ implies that there exist one or more limit cycles. They are located in $\mathcal{D}$ and encircle the points $x_{e 0}, p_{0}$, and $-p_{0}$.

Proof. Case $f_{2}=0$. From Eq. (B.1), the inner product becomes

$$
f \dot{x}\left(x \in \partial \mathcal{L}_{+}\right)=\lambda_{1}\left(1+f_{1}\right)<0,
$$

since the second Hurwitz condition holds: $1+f_{1}+f_{2}=1+f_{1}<0$. Thus, $p_{0}$ does not exist and all trajectories entering the linear region $\mathcal{L}$ cannot leave it. Since the linear region is stable, there is no limit cycle.

Case $f_{2}>0$. Equations (B.1) and (B.2) give

$$
f \dot{x}\left(p_{0}\right)=f_{2}\left(\lambda_{2}-\lambda_{1}\right)\left(1+x_{2}\right)+\lambda_{1}\left(1+f_{1}+f_{2}\right)=0 .
$$

Using the second Hurwitz condition $\lambda_{1}\left(1+f_{1}+f_{2}\right)<0$, we see that the first term of (B.3) satisfies $f_{2}\left(\lambda_{2}-\lambda_{1}\right)\left(1+x_{2}\right)>0$. Moreover, since $f_{2}\left(\lambda_{2}-\lambda_{1}\right)<$ 0 , the expression $\left(1+x_{2}\right)$ is negative, which means that $x_{2}$ of $p_{0}$ satisfies $x_{2}<-1$. Thus, $p_{0}$ is not located in $\mathcal{D}$. Lemma B.1 implies that there cannot exist any limit cycle.

Case $f_{2}<0$. In this case, $f_{2}\left(\lambda_{2}-\lambda_{1}\right)>0$ implies that $x_{2}$ of $p_{0}$ satisfies $x_{2}>-1$. Thus, there exist points $p_{0}$ which are elements of $\mathcal{D}$. This means that one or more limit cycles can occur. Since a limit cycle cannot appear exclusively in the linear region, it has to pass through the positive and negative saturated regions. By Lemma B.2, the trajectory of the limit cycle exits the linear region $\mathcal{L}$ on the open segment $\overline{p_{0} c_{+}} \backslash p_{0} \subset \partial \mathcal{L}_{+}$and reenters $\mathcal{L}$ on the open segment $\overline{p_{0}-r_{-}} \backslash p_{0} \subset \partial \mathcal{L}_{+}$. Owing to the symmetry, the same consideration can be drawn for $\partial \mathcal{L}_{-}$and $-p_{0} \in \partial \mathcal{L}_{-}$. Thus, all limit cycles encircle $p_{0}$ and $-p_{0}$. The fact that the equilibrium point $x_{e 0}$ is encircled is a consequence of the Poincaré theorem. 


\section{Appendix C. Contraction analysis for the trajectories OF SECOND-ORDER LINEAR SYSTEMS}

Define the line $f x=\frac{1}{k}$ (or simply $k f x=1$ ) in the state space, where $x \in \mathbb{R}^{2}$ represents the state variables and where

$$
f=\left[\begin{array}{ll}
f_{1} & f_{2}
\end{array}\right],
$$

$f_{1}, f_{2} \in \mathbb{R}$ and $k \in \mathbb{R} \backslash 0$. Two trajectories starting from the line $k f x=1$ are considered. They evolve until they intersect this same line. The starting points are denoted by $p_{1}$ and $p_{2}$, and the intersection points are denoted by $p_{1}^{\prime}$ and $p_{2}^{\prime}$. Now we consider the distances $d=\left\|p_{1}-p_{2}\right\|$ and $d^{\prime}=\left\|p_{1}^{\prime}-p_{2}^{\prime}\right\|$ on the line $k f x=1$, and the condition under which the distance between the two trajectories decreases, $d^{\prime}<d$, or increases, $d^{\prime}>d$.

C.1. Unstable systems. First, we consider the autonomous second-order unstable system

$$
\dot{x}=A x=\left[\begin{array}{cc}
0 & a_{1} \\
1 & a_{2}
\end{array}\right] x=\left[\begin{array}{cc}
0 & -\lambda_{1} \lambda_{2} \\
1 & \lambda_{1}+\lambda_{2}
\end{array}\right] x, \quad a_{1}>0, \quad a_{2} \in \mathbb{R},
$$

with a positive and a negative real eigenvalues $\lambda(A)=\left\{\lambda_{1}, \lambda_{2}\right\}$ where $\lambda_{1}>0$ and $\lambda_{2}<0$. Without loss of generality, the trajectories with respect to the horizontal line $k f x=1$ is examined, where

$$
f=\left[\begin{array}{ll}
0 & 1
\end{array}\right], \quad k<0 .
$$

On this line $x_{2}=1 / k$ (see Fig. 4 ), we denote the points

$$
\begin{gathered}
p_{m}=\left[\begin{array}{c}
x_{m 1} \\
1 / k
\end{array}\right]=\left[\begin{array}{c}
-\lambda_{1} / k \\
1 / k
\end{array}\right], \quad p_{m}^{\prime}=\left[\begin{array}{c}
y_{m 1} \\
1 / k
\end{array}\right]=\left[\begin{array}{c}
-\lambda_{2} / k \\
1 / k
\end{array}\right], \\
p_{0}=p_{0}^{\prime}=\left[\begin{array}{c}
x_{01} \\
1 / k
\end{array}\right]=\left[\begin{array}{c}
-\left(\lambda_{1}+\lambda_{2}\right) / k \\
1 / k
\end{array}\right] .
\end{gathered}
$$

From now on, $a(>,<,=) 0: b(>,<,=) 0$ means that

(i) if $a>0$, then $b>0$,

(ii) if $a<0$, then $b<0$,

(iii) if $a=0$, then $b=0$.

Lemma C.1. Let $x_{11} \geq x_{01}$ and let $p=\left[\begin{array}{ll}x_{11} & 1 / k\end{array}\right]^{T}$ be a point on the line $k f x=1$. The trajectory $x(t)=e^{A t} p, t \geq 0$, returns to this line if and only if $x_{11}<x_{m 1}$. Let $T$ be the first time of its returning and $p^{\prime}=\left[\begin{array}{ll}y_{11} & 1 / k\end{array}\right]$ be the corresponding intersection point, i.e., $p^{\prime}=e^{A T} p$. This defines two functions, $x_{11} \rightarrow y_{11}$ and $x_{11} \rightarrow T$. Then for all $x_{11} \in$ $\left(x_{01}, x_{m 1}\right)$ and $y_{11} \in\left(y_{m 1}, x_{01}\right)$, we have $\frac{\partial T}{\partial x_{11}}>0$ and $\frac{\partial T}{\partial y_{11}}<0$. Now depending upon whether $\lambda_{1}+\lambda_{2}(>,<,=) 0$, both $\left|y_{11}-x_{01}\right|-\left|x_{11}-x_{01}\right|$ $(>,<,=) 0$ and $\frac{\partial^{2} y_{11}}{\partial x_{11}^{2}}(>,<,=) 0$. Moreover, if $\lambda_{1}+\lambda_{2}>0$, then $\frac{\partial y_{11}}{\partial x_{11}} \in$ 


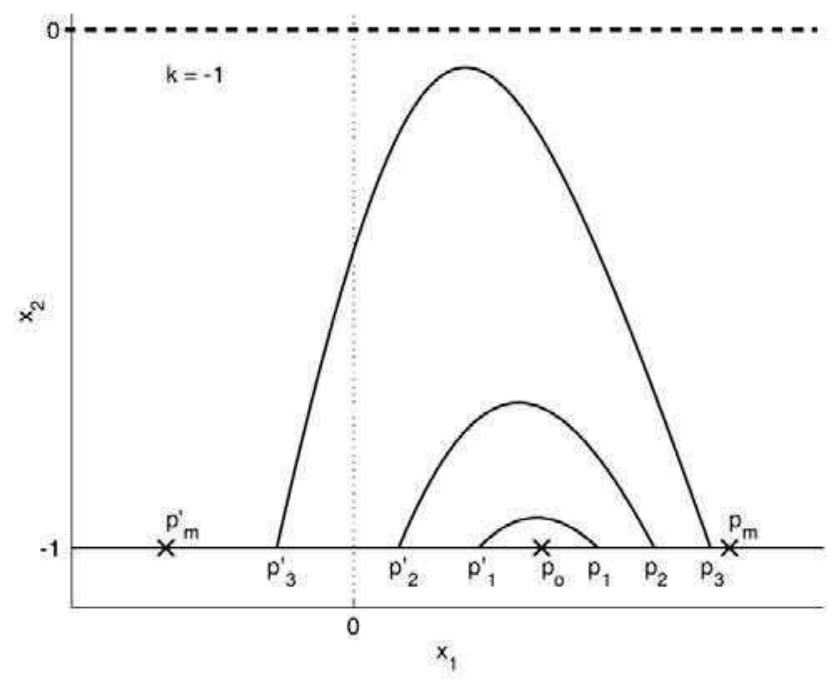

Fig. 4. Illustration of Lemma C.1 for $\lambda_{2}<\lambda_{1}$.

$[-\infty,-1)$. If $\lambda_{1}+\lambda_{2}<0$, then $\frac{\partial y_{11}}{\partial x_{11}} \in(-1,0]$. Finally, if $\lambda_{1}+\lambda_{2}=0$, then $\frac{\partial y_{11}}{\partial x_{11}}=-1$.

Proof. Since at the intersection point $p^{\prime}$, the trajectory goes downward, it follows that $y_{11}<x_{01}$. Using the fact that both $f p=f p^{\prime}=\frac{1}{k}$ and $p^{\prime}=e^{A T} p$, we obtain

$$
\begin{aligned}
& {\left[\begin{array}{ll}
0 & k
\end{array}\right] p^{\prime}=\left[\begin{array}{ll}
0 & k
\end{array}\right] e^{A T}\left[\begin{array}{l}
x_{11} \\
1 / k
\end{array}\right]=1,} \\
& {\left[\begin{array}{ll}
0 & k
\end{array}\right] p=\left[\begin{array}{ll}
0 & k
\end{array}\right] e^{-A T}\left[\begin{array}{l}
y_{11} \\
1 / k
\end{array}\right]=1}
\end{aligned}
$$

Let

and, therefore,

$$
V=\left[\begin{array}{cc}
-\lambda_{2} & -\lambda_{1} \\
1 & 1
\end{array}\right]
$$

Equations (C.2) and (C.3) give:

$$
e^{A T}=V\left[\begin{array}{cc}
e^{\lambda_{1} T} & 0 \\
0 & e^{\lambda_{2} T}
\end{array}\right] V^{-1}
$$

$$
\begin{gathered}
x_{11}(T)=\frac{1}{k} \frac{\lambda_{1}-\lambda_{2}+\lambda_{2} e^{\lambda_{2} T}-\lambda_{1} e^{\lambda_{1} T}}{e^{\lambda_{1} T}-e^{\lambda_{2} T}}, \\
y_{11}(T)=\frac{1}{k} \frac{\lambda_{1}-\lambda_{2}+\lambda_{2} e^{-\lambda_{2} T}-\lambda_{1} e^{-\lambda_{1} T}}{e^{-\lambda_{1} T}-e^{-\lambda_{2} T}} .
\end{gathered}
$$


Owing to the uniqueness of the trajectory, $T$ is also uniquely defined by $x_{11}$. Therefore, $x_{11} \leftrightarrow T, x_{11} \leftrightarrow y_{11}$, and $y_{11} \leftrightarrow T$ are all one-to-one mappings. From the above two equations, $x_{11}(T)$ and $y_{11}(T)$ are analytic on $T \in(0, \infty)$. From Eqs. (C.4) and (C.5), define the gradient

$$
g(T):=-\frac{\partial y_{11}}{\partial x_{11}}=-\frac{\partial y_{11} / \partial T}{\partial x_{11} / \partial T}=\frac{\lambda_{1}-\lambda_{2}-\lambda_{1} e^{\lambda_{2} T}+\lambda_{2} e^{\lambda_{1} T}}{\lambda_{1}-\lambda_{2}-\lambda_{1} e^{-\lambda_{2} T}+\lambda_{2} e^{-\lambda_{1} T}},
$$

where

$$
g(T)>0 \quad \forall T \in(0, \infty) .
$$

After inspecting $g(T)$ for all $T>0$, we obtain:

$$
\begin{array}{ccc}
\lambda_{1}+\lambda_{2}>0: & g(T)>1 & \Longrightarrow \frac{\partial y_{11}}{\partial x_{11}} \in[-\infty,-1) \\
\lambda_{1}+\lambda_{2}<0: & 0 \leq g(T)<1 & \Longrightarrow \frac{\partial y_{11}}{\partial x_{11}} \in(-1,0] \\
\lambda_{1}+\lambda_{2}=0: & g(T)=1 & \Longrightarrow \frac{\partial y_{11}}{\partial x_{11}}=-1 .
\end{array}
$$

Furthermore, since

$$
\frac{\partial g}{\partial T}=\frac{\partial g}{\partial x_{11}} \frac{\partial x_{11}}{\partial T}=-\frac{\partial^{2} y_{11}}{\partial x_{11}^{2}} \frac{\partial x_{11}}{\partial T}, \quad \frac{\partial x_{11}}{\partial T}>0,
$$

it follows that

$$
\lambda_{1}+\lambda_{2}(>,<,=) 0: \quad \frac{\partial g}{\partial T}(>,<,=) 0 \Longrightarrow \frac{\partial^{2} y_{11}}{\partial x_{11}^{2}}(<,>,=) 0 .
$$

From $\lim _{T \rightarrow 0} g(T)=1$ it follows that

$$
\lim _{T \rightarrow 0} \frac{\partial y_{11}}{\partial x_{11}}=-1
$$

and (C.6) becomes:

$$
\lambda_{1}+\lambda_{2}(>,<,=) 0: \quad\left|y_{11}-x_{01}\right|(>,<,=)\left|x_{11}-x_{01}\right| .
$$

The lemma is proved.

Figure 4 illustrates Lemma C. 1 for the case where $\lambda_{1}+\lambda_{2}>0$ and $p_{1}$, $p_{2}$, and $p_{3}$ are three points on $k f x=1$ :

$$
p_{i}=\left[\begin{array}{l}
x_{11}^{i} \\
1 / k
\end{array}\right], \quad x_{11}^{i} \in\left[x_{01}, x_{m 1}\right), \quad x_{11}^{i+1}>x_{11}^{i}, \quad i=1,2,3 .
$$

Comparing Fig. 4 with the content of the lemma, it can be noted that

$$
\lambda_{1}+\lambda_{2}(>,<,=) 0: \quad \frac{\left\|p_{3}^{\prime}-p_{2}^{\prime}\right\|}{\left\|p_{3}-p_{2}\right\|}(>,<,=) \frac{\left\|p_{2}^{\prime}-p_{1}^{\prime}\right\|}{\left\|p_{2}-p_{1}\right\|}(>,<,=) 1 .
$$

Remark. Lemma C.1 is given for the line $k f x=1$, where $k<0$. Since system (C.1) is symmetric, the contraction results still hold for $k f x=-1$, $k<0$. 
Consider any state transformation $x=T \hat{x}$ resulting in

$$
\dot{\hat{x}}=\hat{A} \hat{x}, \quad \hat{A}=T^{-1} A T, \quad k \hat{f}=k f T .
$$

Lemma C.2. Let $k \hat{f} \hat{x}=1$ be chosen, where $k \in \mathbb{R} \backslash 0$. Consider two trajectories $\operatorname{Trj}\left(\hat{p}_{1}, \hat{p}_{1}^{\prime}\right)$ and $\operatorname{Trj}\left(\hat{p}_{2}, \hat{p}_{2}^{\prime}\right)$ starting from $\hat{p}_{1}$ and $\hat{p}_{2}$ on $k \hat{f} \hat{x}=1$ and ending at $\hat{p}_{1}^{\prime}$ and $\hat{p}_{2}^{\prime}$ on $k \hat{f} \hat{x}=1$. Then

$$
\text { for } \lambda_{1}+\lambda_{2}(>,<,=) 0: \quad\left\|\hat{p}_{2}^{\prime}-\hat{p}_{1}^{\prime}\right\|(>,<,=)\left\|\hat{p}_{2}-\hat{p}_{1}\right\| .
$$

Proof. The proof is given in [6].

C.2. Stable systems. Consider the autonomous, second-order stable system

$$
\dot{x}=A x=\left[\begin{array}{ll}
0 & -a_{1} \\
1 & -a_{2}
\end{array}\right] x, \quad a_{1}, a_{2}>0,
$$

where $a_{1}=\lambda_{1} \lambda_{2}, a_{2}=-\left(\lambda_{1}+\lambda_{2}\right), \operatorname{Re}\left(\lambda_{1}\right)<0$, and $\operatorname{Re}\left(\lambda_{2}\right)<0$. Without loss of generality, two horizontal lines $k f x=1$ and $k f x=-1$ are considered:

$$
f=\left[\begin{array}{ll}
0 & 1
\end{array}\right], \quad k>0 .
$$

For some points on the line $k f x=1$, the trajectories of system (C.8) starting from these points enter the region

$$
\left\{x \in \mathbb{R}^{2}:|k f x|<1\right\}
$$

and then intersect the line $k f x=-1$. Let

$$
p_{0}=\left[\begin{array}{c}
y_{m 2} \\
-1 / k
\end{array}\right]=\left[\begin{array}{c}
-a_{2} / k \\
-1 / k
\end{array}\right]=\left[\begin{array}{c}
\left(\lambda_{1}+\lambda_{2}\right) / k \\
-1 / k
\end{array}\right]
$$

be a point on $k f x=-1$. If a point $x$ is on $k f x=-1$ and lies on the left of $p_{0}\left(x_{1}<y_{m 2}\right)$, then the vector $\dot{x}$ points downward and $\dot{x}_{2}<0$; if $x$ lies to right of $p_{0}\left(x_{1}>y_{m 2}\right)$, then the vector $\dot{x}$ points upward and $\dot{x}_{2}>0$ (see Fig. 5).

Definition C.3. Let $p_{0}^{\prime}$ be the unique point on $k f x=1$ and $T_{d}>0$ be the unique number satisfying

$$
e^{A T_{d}} p_{0}^{\prime}=p_{0}, \quad\left|k f e^{A t} p_{0}^{\prime}\right| \leq 1 \quad \forall t \in\left[0, T_{d}\right] .
$$

Denote the first coordinate of $p_{0}^{\prime}$ by $x_{m 2}$ :

$$
p_{0}^{\prime}=\left[\begin{array}{c}
x_{m 2} \\
1 / k
\end{array}\right] .
$$

For $x_{11} \in\left(-\infty, x_{m 2}\right]$, let

$$
p^{\prime}=\left[\begin{array}{l}
x_{11} \\
1 / k
\end{array}\right]
$$

be a point on $k f x=1$. Then there exists

(i) a unique $p=\left[\begin{array}{ll}y_{11} & -1 / k\end{array}\right]$ on $k f x=-1$, where $y_{11} \in\left(-\infty, y_{m 2}\right]$; 


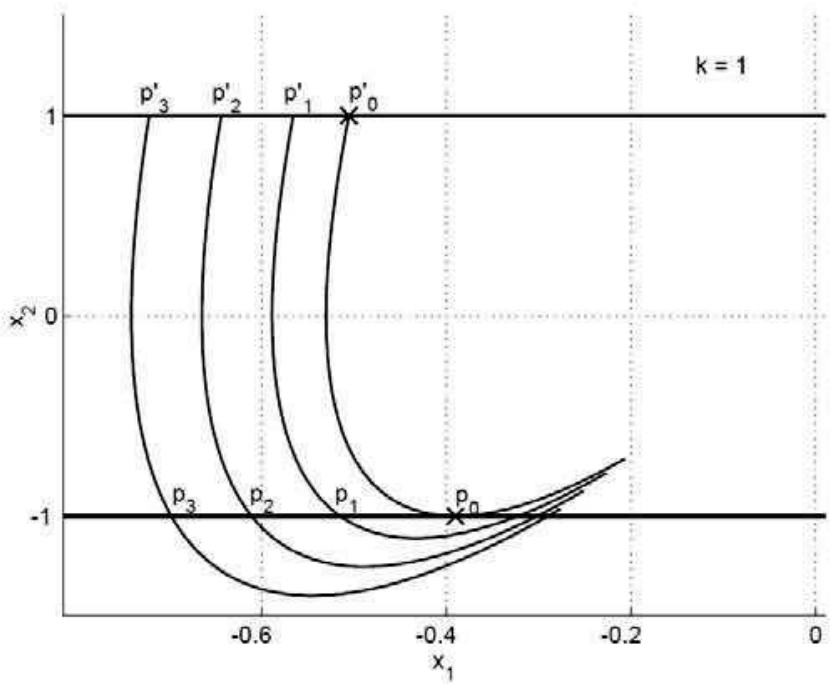

Fig. 5. Illustration of Lemma C.4.

(ii) a unique $T \in\left(0, T_{d}\right]$ such that

$$
p=e^{A T_{d}} p^{\prime}, \quad\left|k f e^{A t} p^{\prime}\right| \leq 1 \quad \forall t \in[0, T] .
$$

This defines two functions $x_{11} \rightarrow y_{11}$ and $x_{11} \rightarrow T$ with $x_{11} \in\left(-\infty, x_{m 2}\right]$, $y_{11} \in\left(-\infty, y_{m 2}\right]$, and $T \in\left(0, T_{d}\right]$.

Lemma C.4. For all $x_{11} \in\left(-\infty, x_{m 2}\right)$, we have $x_{11}<y_{11}$ and

$$
\frac{\partial y_{11}}{\partial x_{11}}>1, \quad \frac{\partial^{2} y_{11}}{\partial x_{11}^{2}}>0, \quad \frac{\partial T}{\partial x_{11}}>0 .
$$

Proof. The proof is given in $[6,8]$.

This lemma is illustrated in Fig. 5, where $p_{1}^{\prime}, p_{2}^{\prime}$, and $p_{3}^{\prime}$ are three points on $k f x=1$ and $p_{1}, p_{2}$, and $p_{3}$ are the first three intersections of $k f x=-1$ with three trajectories starting from $p_{1}^{\prime}, p_{2}^{\prime}$, and $p_{3}^{\prime}$, respectively. Then, by Lemma C.4, we can conclude that

$$
\frac{\left\|p_{1}-p_{2}\right\|}{\left\|p_{1}^{\prime}-p_{2}^{\prime}\right\|}>\frac{\left\|p_{2}-p_{3}\right\|}{\left\|p_{2}^{\prime}-p_{3}^{\prime}\right\|}>1
$$

Lemma C.5. Consider the planar stable system $\dot{\hat{x}}=\hat{A} \hat{x}$ and the lines $k \hat{f} \hat{x}=1$ and $k \hat{f} \hat{x}=-1$, where $k \in \mathbb{R} \backslash 0$, can be arbitrary chosen. Moreover, consider two trajectories $\operatorname{Trj}\left(\hat{p}_{1}^{\prime}, \hat{p}_{1}\right) \subset \mathcal{L}$ and $\operatorname{Trj}\left(\hat{p}_{2}^{\prime}, \hat{p}_{2}\right) \subset \mathcal{L}$, where 
the trajectories start from $\hat{p}_{1}^{\prime}$ and $\hat{p}_{2}^{\prime}$ on $k \hat{f} \hat{x}=1$ and end at $\hat{p}_{1}$ and $\hat{p}_{2}$ on $k \hat{f} \hat{x}=-1$. Then the condition

$$
\left\|\hat{p}_{1}-\hat{p}_{2}\right\|>\left\|\hat{p}_{1}^{\prime}-\hat{p}_{2}^{\prime}\right\|
$$

holds.

Proof. The proof is given in [6].

\section{REFERENCES}

1. J. Alvarez, R. Suárez, and J. Alvarez, Planar linear systems with single saturated feedback. Systems Control Lett. 20 (1993), 319-326.

2. U. Boscain and B. Piccoli, Extremal syntheses for generic planar systems. J. Dynam. Control Systems 7 (2001), No. 2, 209-258.

3. __ Optimal synthesis for control systems on 2-D manifolds. Springer-Verlag, Berlin (2004).

4. A. Bressan and B. Piccoli, A generic classification of time optimal planar stabilizing feedbacks. SIAM J. Control Optimiz. 36 (1998), No. 1, 1232.

5. $\quad$ Structural stability for time-optimal planar syntheses. Dynam. Contin. Discr. Impuls. Systems 3 (1997), No. 3, 335-371.

6. J.-Y. Favez, Enhancing the control of tokamaks via a continuous nonlinear control law. Ph.D. thesis, No. 3034, École Polytechnique Fédérale de Lausanne, Switzerland (2004), http://library.epfl.ch/theses.

7. J.-Y. Favez, B. Srinivasan, P. Mullhaupt, and D. Bonvin, Condition for bifurcation of the region of attraction in linear planar systems with saturated linear feedback. In: 41th Conf. on Decision and Control, Las Vegas (2002), pp. 3918-3923.

8. T. Hu and Z. Lin, Control systems with actuator saturation: Analyses and design. Birkhäuser, Boston (2000).

9. T. Hu, Z. Lin, and L. Qiu, Stabilization of exponentially unstable linear systems with saturating actuators. IEEE Trans. Automat. Control 46 (2001), No. 6, 973-979.

10. H. K. Khalil, Nonlinear systems. Prentice-Hall (1996).

11. Z. Lin and A. Saberi, Semi-global exponential stabilization of linear systems subject to input saturation via linear feedback. Systems Control Lett. 21 (1993), 225-239.

12. L. Scibile and B. Kouvaritakis, Stability region for a class of open-loop unstable linear systems: theory and application. Automatica 36 (2000), $37-44$.

13. J.-J. E. Slotine and W. Li, Applied nonlinear control. Prentice-Hall (1991).

14. H. J. Sussmann, The structure of time-optimal trajectories for singleinput systems in the plane: the general real analytic case. SIAM J. Control Optim. 25 (1987), No. 4, 868-904. 
15. analytic systems in the plane. SIAM J. Control Optim. 25 (1987), No. 5, $1145-1162$.

16. H. J. Sussmann, E. D. Sontag, and Y. Yang, A general result on the stabilization of linear systems using bounded controls. IEEE Trans. Automat. Control 39 (1994), No. 12, 2411-2424.

(Received March 03 2005, received in revised form October 24 2005)

Authors' addresses:

J.-Y. Favez

Laboratoire d'Automatique,

Ecole Polytechnique Fédérale de Lausanne,

CH-1015 Lausanne, Switzerland

Ph. Mullhaupt

Laboratoire d'Automatique,

Ecole Polytechnique Fédérale de Lausanne,

CH-1015 Lausanne, Switzerland

E-mail: philippe.muellhaupt@epfl.ch

B. Srinivasan

Ecole Polytechnique Montréal, Génie Chimique,

C.P. 6079, Succ. Centre-Ville, Montréal, Canada

D. Bonvin

Laboratoire d'Automatique,

Ecole Polytechnique Fédérale de Lausanne,

CH-1015 Lausanne, Switzerland 